\title{
Photoresponse of Solution-Processed Molybdenum Disulfide Nanosheet-Based Photodetectors
}

Vijith K. Pulikodan, Akhil Alexander, Anitha B. Pillai, Manoj A. G. Namboothiry*

School of Physics,

Indian Institute of Science Education and Research Thiruvananthapuram (IISER-TVM), Maruthamala PO, Vithura, Thiruvananthapuram, Kerala, 695551, India.

E-mail: manoj@iisertvm.ac.in
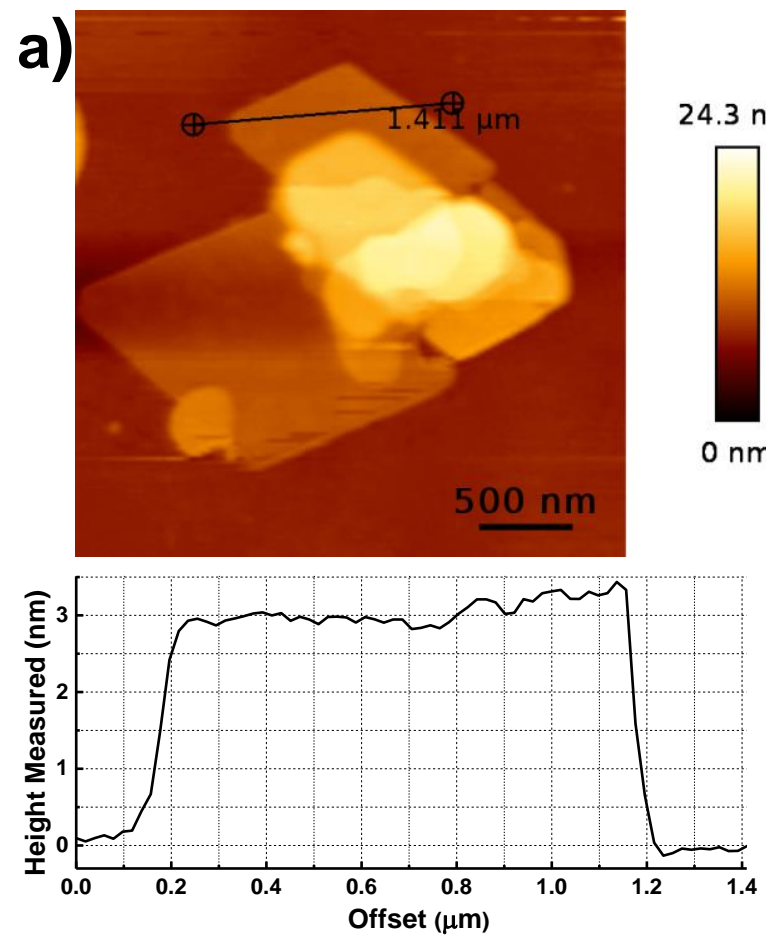

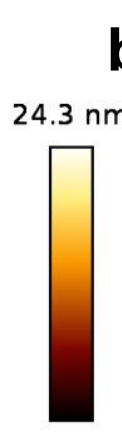

$0 \mathrm{~nm}$

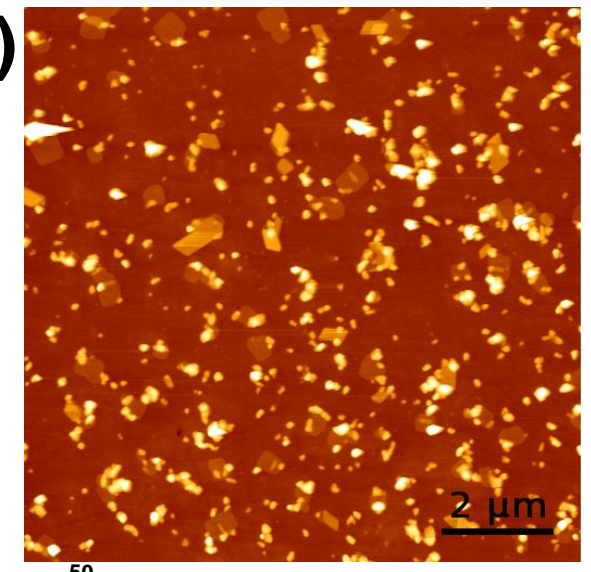

$17.6 \mathrm{~nm}$

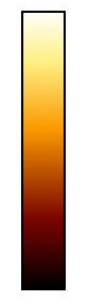

$0 \mathrm{~nm}$

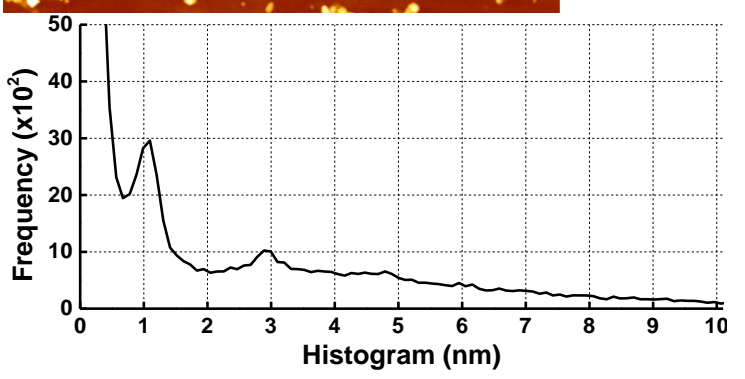

Figure S1. Atomic force microscopy of exfoliated $\mathrm{MoS}_{2} \mathrm{NSs}$ with height profile and histogram. In the histogram, large peak around zero is due to the height variations from the substrate and the peaks centered at $\sim 1.3$ and $\sim 2.8 \mathrm{~nm}$ are from the height distribution of NSs. 


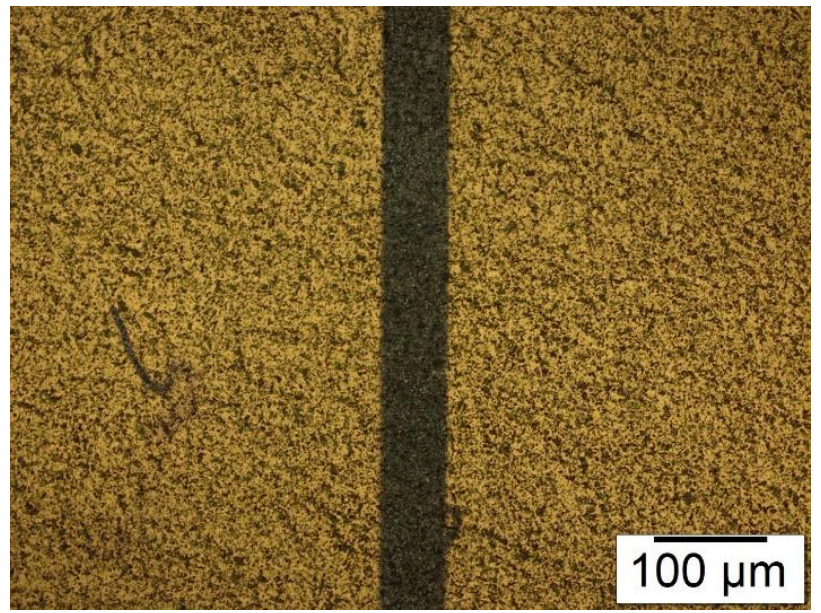

Figure S2. Optical micrograph of $\mathrm{MoS}_{2}$ photodetector showing device area and gold contacts. The channel length of the device is fixed at $50 \mu \mathrm{m}$.
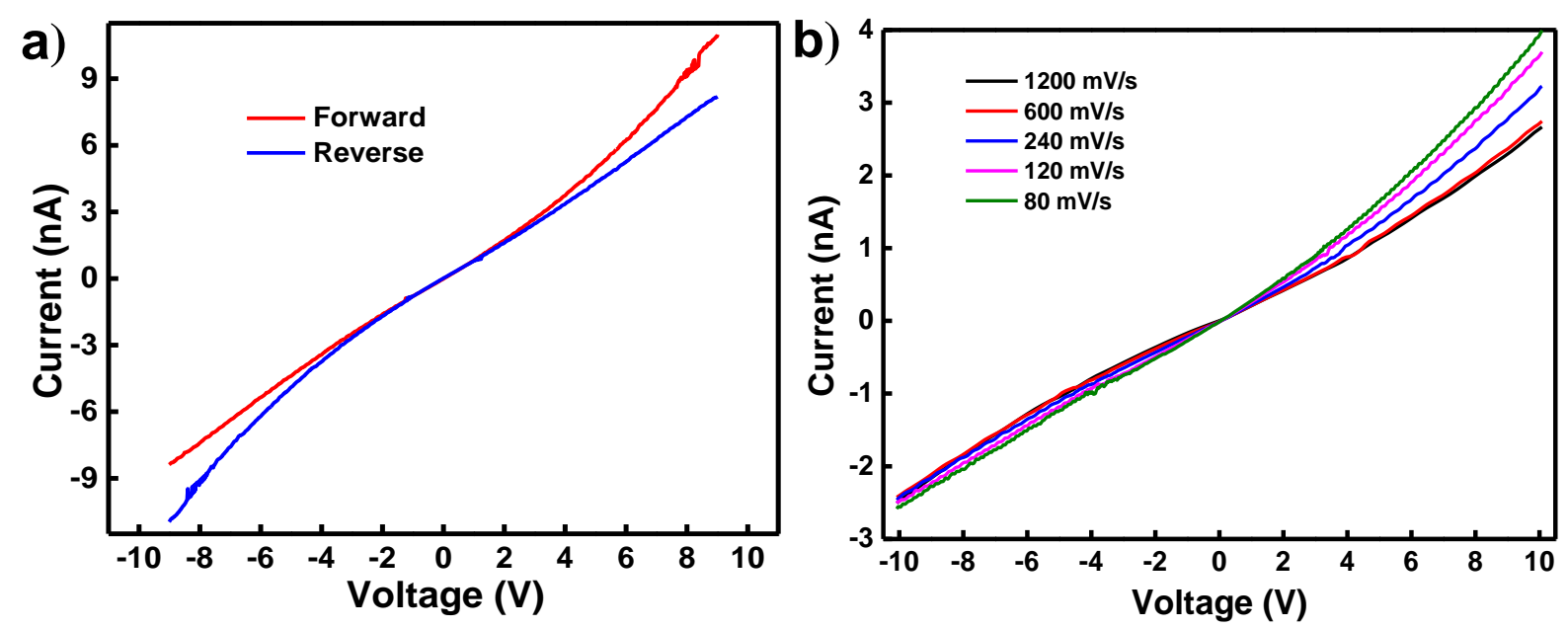

Figure S3. I- $V$ measurement of $\mathrm{MoS}_{2}$ photodetector under illumination (a) during forward (red color) and reverse (blue color) scanning and (b) for different scanning speed of bias voltage. 
Specific detectivity $\left(D^{*}\right)$ of the $\mathrm{MoS}_{2}$ photodetectors estimated using shot noise limited specific detectivity equation,

$$
D^{*}=\frac{R \sqrt{A}}{\sqrt{2 q I_{d}}}
$$

where, $R, A$ and $I_{d}$ are the responsivity, effective area and dark current of the photoconductor respectively and q represents the elementary charge of electron.

Noise equivalent power $(N E P)$ of the detector is calculated using the equation,

$$
N E P=\frac{\sqrt{2 q I_{d} \Delta f}}{R}
$$

where $\Delta f$ is taken as 1 .

a)

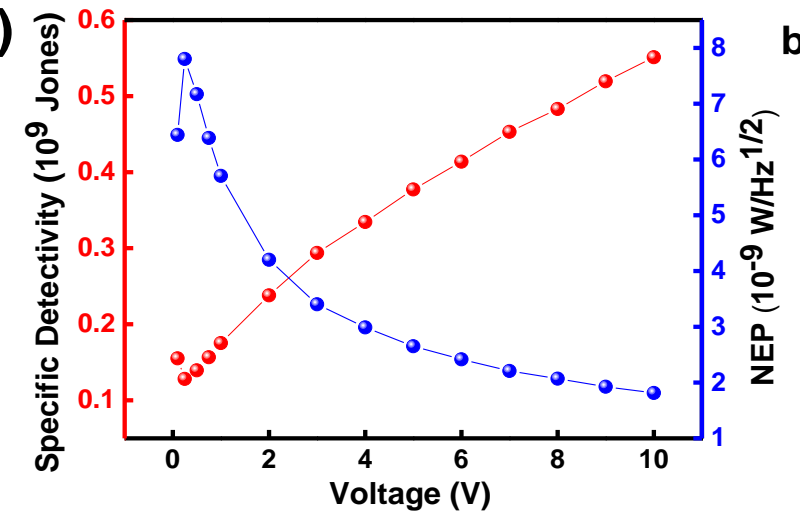

b)

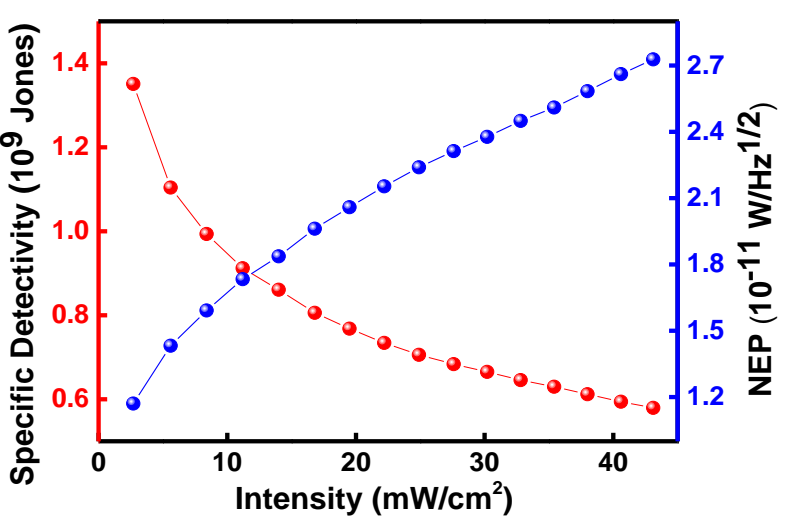

Figure S4. Variation in short noise limited specific detectivity and noise equivalent power of the $\mathrm{MoS}_{2}$ photodetectors with a) increasing bias voltage and b) increasing illumination intensity.

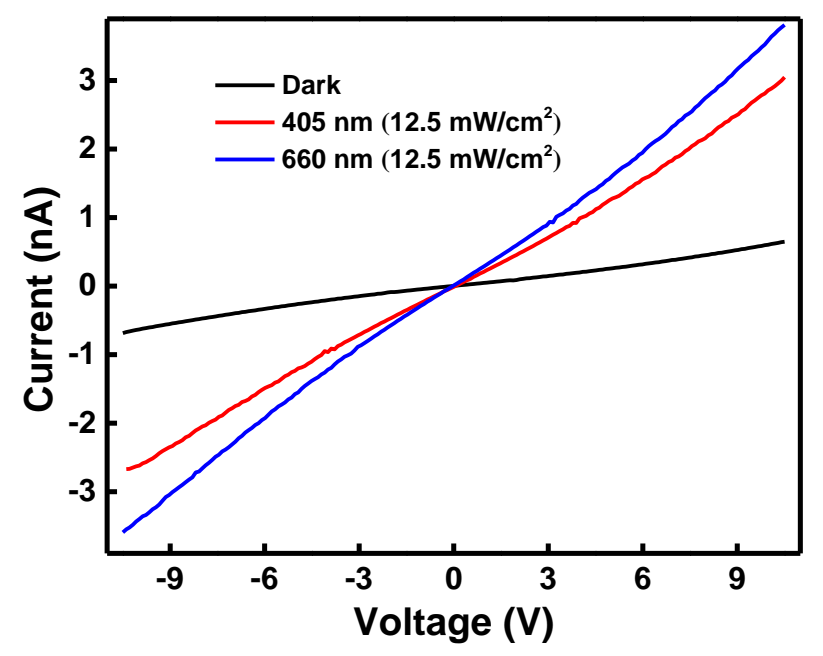

Figure S5. I- $V$ measurement of $\mathrm{MoS}_{2}$ photodetector in dark (black color) and under illumination with two different wavelength of light with $12.5 \mathrm{~mW} \mathrm{~cm}^{-2}$ illumination intensity. 